\title{
Remarks On The Spherical Scalar Field Halo In Galaxies
}

\author{
Kamal K. Nandi ${ }^{1,2,3, a}$, Ildar Valitov ${ }^{2, b}$ and Nail G. Migranov ${ }^{3, c}$ \\ ${ }^{1}$ Department of Mathematics, University of North Bengal, Siliguri 734013 , \\ India \\ ${ }^{2}$ Department of Theoretical Physics, Sterlitamak State Pedagogical Academy, \\ Sterlitamak 453103, Russia \\ ${ }^{3}$ Joint Research Laboratory, Bashkir State Pedagogical University, Ufa 450000, \\ Russia \\ ${ }^{a}$ E-mail: kamalnandi1952@yahoo.co.in \\ ${ }^{b}$ E-mail: diamond921@yandex.ru \\ ${ }^{c}$ E-mail: ufangm@yahoo.co.uk
}

\begin{abstract}
Matos, Guzmán and Nuñez proposed a model for the galactic halo within the framework of scalar field theory. We argue that an analysis involving the full metric can reveal the true physical nature of the halo only when a certain condition is maintained. We fix that condition and also calculate its impact on observable parameters of the model.
\end{abstract}

One of the outstanding problems in modern astrophysics is the problem of dark matter which is invoked as an explanation for the observed flat rotation curves in the galactic halo. Doppler emissions from stable circular orbits of neutral hydrogen clouds in the halo allow the measurement of tangential velocity $v_{t g}(r)$ of the clouds treated as probe particles. According to Newton's laws, centrifugal acceleration $v_{t g}^{2} / r$ should balance the gravitational attraction $G M(r) / r^{2}$, which immediately gives $v_{t g}^{2}=G M(r) / r$. That is, one would expect a fall-off of $v_{t g}^{2}(r)$ with $r$. However, observations indicate that this is not the case: $v_{t g}$ approximately levels off with $r$ in the halo region. The only way to interpret this result of observation is to accept that the mass $M(r)$ increases linearly with distance $r$. Luminous mass distribution in the galaxy does not follow this behavior. Hence the hypothesis that there must be huge amounts of nonluminous matter hidden in the halo. This unseen matter is given a technical name dark matter.

Despite the fact that the exact nature of dark matter is as yet unknown, several analytic halo models exist in the literature including those provided by scalar-tensor theories (see for instance [1]). In particular, the scalar field model first proposed by Matos, Guzmán and Nuñez [2] has received considerable attention. It is important to note that the authors primarily constructed an exact solution of Einstein's field equations sourced by a scalar field that provides a density profile of $1 / r^{2}$ together with other appealing features of the metric 
functions. As a particular application, they sketched a plausible interpretation of the halo dark matter problem. The problem being important in itself, we think that the interesting relativistic central feature of the solution, namely, a non-Newtonian halo, must be well grounded. The purpose of the present Brief Report is to fix the condition under which it is possible. In addition, we work out its impact on observable parameters.

It is to be mentioned that the solution in [2] has been criticized because of its singular behavior at the origin [3], but this singularity is not peculiar to that solution alone; there are other viable halo models in the literature that also possess such singularity (see for instance [4]). While we are here interested only in the outer reaches of the galaxy (very far from the origin), the authors of Ref.[2] and the associated research group have addressed this inner region singularity too. They obtained several new results under the scalar field dark matter model in galaxies: Solution with axial symmetry including the inner region [5], timedependent spacetimes [6], the full non-linear Newtonian evolution after the turnaround point [7], time evolution of density fluctuation [8], collision properties of two structures [9] and so on. While they obtained constraints arising out of cosmological scale considerations, we believe that it is also useful to ascertain the constraint arising out of the local scale, which would clarify the relativistic nature of the spherically symmetric model of the halo under consideration.

Using the flat rotation curve condition [10], Matos, Guzmán and Nuñez obtain the spherically symmetric static solution for the galactic halo as follows ( $G=c=1$, unless specifically restored):

$$
\begin{gathered}
d s^{2}=-B(r) d t^{2}+A(r) d r^{2}+r^{2}\left(d \theta^{2}+\sin ^{2} \theta d \phi^{2}\right) \\
B(r)=B_{0} r^{l} \\
A(r)=\frac{4-l^{2}}{4+D\left(4-l^{2}\right) r^{-(l+2)}} \\
\phi(r)=\sqrt{\frac{l}{8 \pi}} \ln r+\phi_{0} \\
V(r)=-\frac{1}{8 \pi(2-l) r^{2}},
\end{gathered}
$$

where $D$ is an arbitrary constant of integration, $\phi$ and $V$ are the scalar field and potential respectively. The parameter $l=2\left(v_{t g} / c\right)^{2}, B_{0}>0$ is another constant. Observations of the frequency shifts in the HI radiation show that, in the halo region, $v_{t g} / c$ is nearly constant at a value $7 \times 10^{-4}[11]$. Thus, in what follows, we take $l \sim 10^{-6}$.

Note that we can rewrite $A(r)$ in the standard Schwarzschild form

$$
A(r)=\left[1-\frac{2 m(r)}{r}\right]^{-1}
$$

which is often convenient and will be useful later while discussing the observational parameters. Such a form has the advantage that it immediately reveals 
not only the mass parameter $m(r)$ but also shows that the proper radial length is larger than the Euclidean length because $r>2 m(r)$. This inequality, which is essential for signature protection, dictates that $A(r)>1$. This is a crucial condition to be satisfied by any valid metric.

Now, for the sake of simplicity, Matos, Guzmán and Nuñez choose $D=0$, but this is not the best choice because it makes the metric component $A<1$. As a consequence, whatever results follow from the reduced metric should be taken with caution. For instance, the stresses exhibit a density profile $\rho<0$, meaning violation of Weak Energy Condition (WEC) and furthermore lead to $\omega<-1$ (see below), meaning repulsive gravity in the halo, contradicting observational facts. But these are actually not the true features of their model. To see the true picture, it is necessary to calculate the relevant quantities with $D \neq 0$.

We find the density and pressure profiles in the rest frame of the fluid as

$$
\begin{gathered}
\rho=\frac{1}{8 \pi} \frac{r^{-(4+l)}\left[D\left(l^{3}+l^{2}-4 l-4\right)+l^{2} r^{2+l}\right]}{l^{2}-4} \\
p_{r}=\frac{1}{8 \pi} \frac{r^{-(4+l)}\left[D\left(l^{3}+l^{2}-4 l-4\right)-l(4+l) r^{2+l}\right]}{l^{2}-4} \\
p_{t}=\frac{1}{8 \pi} \frac{r^{-(6+l)}\left[D\left(l^{3}+l^{2}-4 l-4\right)+l^{2} r^{2+l}\right]\left[\left(r^{2}-1\right) l-2\left(r^{2}+1\right)\right]}{4\left(l^{2}-4\right)}
\end{gathered}
$$

where $\rho$ is the energy density, $p_{r}$ is the radial pressure and $p_{t}$ are the transverse pressures.

Matos, Guzmán and Nuñez conclude that their model has huge pressure over density and thus it is non-Newtonian. We wish to emphasize that the role of nonzero value of $D$ is crucial not only for avoiding repulsive gravity (as alluded to above) but also for arriving at a correct conclusion about the relative strengths between pressure and density. For instance, let us take $D=1$. In the distant halo region, we can take, typically, $r \sim 100-300 K p c$ and with $l \sim 10^{-6}$, we find the numerical values to be $\rho \sim 10^{-9}$ and $p_{r} \sim 10^{-9}$, which means that they are of the same order. But on the other hand, $p_{r}+2 p_{t} \sim 10^{-11} \Rightarrow p_{r}+2 p_{t} \sim 10^{-2} \rho$, which indicates that total pressure is roughly one hundred times less than the density. However, if we take $D=10^{-5}$, we find that $p_{r}+2 p_{t} \sim 10^{3} \rho$. If we keep on decreasing the value of $D$ further (but never exactly to zero for reasons stated above), we see that the total pressure dominates more and more over density reinforcing the non-Newtonian nature.

The next question is: How far can we go on decreasing $D$ ? We notice the following interesting scenario. When $D=10^{-7}$, we find $p_{r}+2 p_{t}=9 \times 10^{5} \rho$, which leads to $\omega=\frac{p_{r}+2 p_{t}}{3 \rho}=3 \times 10^{5}$ (attractive gravity) as shown in Fig.1. This is the extreme possible non-Newtonian halo in the scalar field model under consideration. The reason is this. If $D=10^{-8}$, we find that $\omega>0$ up to $r=r_{0}=200 K p c$ (attractive gravity) and becomes $\omega<-1$ after $r=r_{0}$ (repulsive gravity). At $r=r_{0}$, there is a singularity in $\omega$. This value of $D$ represents a transition from attraction to repulsion as shown in Fig.2. When $D \leq 10^{-9}$, we find that $\rho<0, \omega<-1$ (repulsive gravity), which share the 
woes that follow also from the choice $D=0$ (Figs.3 and 4). These show that we can not decrease $D$ below $10^{-7}$, that is, we must have $D \geq 10^{-7}$. This is the condition that must be maintained in order to have a non-Newtonian halo.

The pressures are anisotropic, as is evident from Eqs. $(7,8)$, which is a good feature of the solution from the point of view of exterior matching. Note that the solution can not be matched to the Schwarzschild exterior metric at the boundary of the halo if the pressures were isotropic [12]. It can be further verified that $\rho>0, \rho+p_{r}>0, \rho+p_{r}+2 p_{t}>0$ for $D \geq 10^{-7}$; so we can say that the halo matter is not exotic because the standard energy conditions are satisfied everywhere. Therefore, we expect attractive gravity in the halo. To confirm it, we follow the prescription by Lynden-Bell, Katz and Bičák [13], and find that the total gravitational energy is indeed negative:

$$
E_{G}=4 \pi \int_{r_{1}}^{r_{2}}\left[1-A^{\frac{1}{2}}\right] \rho r^{2} d r<0,
$$

due to the fact that $\rho>0,1-A^{\frac{1}{2}}<0$ and $r_{2}>r_{1}$. This prescription has been very useful in the case of scalar field wormholes too [14-16].

Certainly, the scalar field model corresponding to $D=10^{-7}$ is highly nonNewtonian because $p_{r}+2 p_{t} \sim 10^{6} \rho$. As a result, a purely Newtonian definition of mass, viz., $M(r)=4 \pi \int \rho r^{2} d r$ does not apply. However, incorporating the pressure contribution, the dynamical mass in the first post Newtonian order becomes

$$
M_{p N}(r)=4 \pi \int\left(\rho+p_{r}+2 p_{t}\right) r^{2} d r=10^{6} M(r),
$$

which clearly reflects the non-Newtonian nature of the model in terms of masses.

We next focus on the observable parameters expected in this non-Newtonian halo. Whatever be the analytic model for it, there must be a way to contrast its predictions with actual measurements. The key point is that one does not directly measure the metric functions but indirectly measures gravitational potentials and masses from rotation curve and lensing observations. Faber and Visser [17] have shown how, in the first post Newtonian approximation, the combined measurements of rotation curves and gravitational lensing allow inferences about the mass and pressure profile of the galactic halo as well as its equation of state.

The usual techniques for obtaining the potential for rotation curve (RC) measurements yield a pseudo-potential (See Ref.[17] for details):

$$
\Phi_{\mathrm{RC}}=\Phi \neq \Phi_{N}
$$

where $\Phi_{N}$ is the Newtonian potential, $\Phi=\frac{1}{2} \ln B$ and a pseudo-mass

$$
m_{\mathrm{RC}}=r^{2} \Phi^{\prime}(r) \approx 4 \pi \int\left(\rho+p_{r}+2 p_{t}\right) r^{2} d r .
$$

Faber and Visser also define the lensing pseudo-potential as

$$
\Phi_{\mathrm{lens}}=\frac{\Phi(r)}{2}+\frac{1}{2} \int \frac{m(r)}{r^{2}} d r .
$$


and a pseudo-mass $m_{\text {lens }}$ obtained from lensing measurements as

$$
m_{\mathrm{lens}}=\frac{1}{2} r^{2} \Phi^{\prime}(r)+\frac{1}{2} m(r)
$$

The first order approximations of Einstein's equations yield

$$
\begin{gathered}
\rho(r) \approx \frac{1}{4 \pi r^{2}}\left[2 m_{\mathrm{lens}}^{\prime}(r)-m_{\mathrm{RC}}^{\prime}(r)\right] \\
4 \pi r^{2}\left(p_{r}+2 p_{t}\right) \approx 2\left[m_{\mathrm{RC}}^{\prime}(r)-m_{\text {lens }}^{\prime}(r)\right]
\end{gathered}
$$

where the right hand sides denote pseudo-density and pseudo-pressures. Furthermore, Faber and Visser define a dimensionless quantity

$$
\omega(r)=\frac{p_{r}+2 p_{t}}{3 \rho} \approx \frac{2}{3} \frac{m_{\mathrm{RC}}^{\prime}-m_{\mathrm{lens}}^{\prime}}{2 m_{\mathrm{lens}}^{\prime}-m_{\mathrm{RC}}^{\prime}}
$$

The pseudo quantities on the right hand side of Eqs.(11)-(17) are actual observables from the combined measurement. If the observed pseudo profiles reasonably match with the analytic pseudo profiles coming from a priori given metric functions, one can say that the solution is physically substantiated. Otherwise, it has to be ruled out as non-viable. The impact of a small non-zero $D$ on the analytic pseudo profiles can now be computed. For the extreme $\left(D=10^{-7}\right)$ Matos, Guzmán and Nuñez solution, these work out to leading order in $r$ as

$$
\begin{gathered}
m_{\mathrm{RC}}(r)=\frac{l r}{2} \approx 10^{-6} r \\
m_{\mathrm{lens}}(r) \approx \frac{l\left(l^{2}+l-4\right) r}{4\left(l^{2}-4\right)} \approx 10^{-6} r \\
2\left(m_{\mathrm{RC}}^{\prime}-m_{\mathrm{lens}}^{\prime}\right) \approx \frac{l\left(l^{2}-l-4\right)}{2\left(l^{2}-4\right)} \approx 10^{-6} .
\end{gathered}
$$

The dimensionless parameter $\omega$ to all orders in $r$ with no restriction on $D$ is

$$
\omega(r) \approx \frac{2}{3} \frac{m_{\mathrm{RC}}^{\prime}-m_{\mathrm{lens}}^{\prime}}{2 m_{\mathrm{lens}}^{\prime}-m_{\mathrm{RC}}^{\prime}}=\frac{l\left(l^{2}-l-4\right) r^{2+l}-D\left(l^{3}+l^{2}-4 l-4\right)}{3\left[D\left(l^{3}+l^{2}-4 l-4\right)+l^{2} r^{2+l}\right]},
$$

which yields $\omega \approx 3 \times 10^{5}$ for $D=10^{-7}$ within our chosen range, $r \sim 100-300$ $K p c$. Note that if we straightaway put $D=0$ in Eq.(21), we get $\omega(r)<-1$, conveying a completely wrong physical conclusion.

The pivotal result of the present article is that $D \geq 10^{-7}$ and not $D=0$, as discussed above. Of course, the lowest limit on $D$ is small and it is quite tempting to set it exactly to zero. But the price for it is that one gets a completely wrong picture of the halo. We have analyzed the model taking into account only the lowest value of $D$. Similar analysis can be carried out with other values of $D$ as well respecting the suggested lower limit. We can say that, by and large, the conclusion of Matos, Guzmán and Nuñez about non-Newtonian nature of the halo is right provided the restriction on $D$ is maintained. With 
this restriction in place, their model can indeed be a physically viable one. If combined measurements follow the pattern as indicated in Eqs.(18)-(21), we would say that the model is observationally supported. However, given the present uncertainties in observation, it is yet too premature to say so.

We are deeply indebted to Guzel N. Kutdusova for her assistance at SSPA and BSPU where the work was carried out.

\section{Figure captions:}

Fig.1. Plot of $\omega(r)$ vs $r$ in which $\omega$ is computed from either Eqs.(6)-(8) or (21) with $l=10^{-6}$ and $D=10^{-7}$. The distance $r$ in galactic halo region is taken in the range $100-300 \mathrm{Kpcs}$. The non-Newtonian values of $\omega$ are evident.

Fig.2. Plot of $\omega(r)$ vs $r$ in which $\omega$ is computed from either Eqs.(6)-(8) or (21) with $l=10^{-6}$ and $D=10^{-8}$. The distance $r$ in galactic halo region is taken in the range $100-300 \mathrm{Kpcs}$. The figure displays the transition behavior of $\omega$ as discussed in the text.

Fig.3. Plot of $\omega(r)$ vs $r$ in which $\omega$ is computed from either Eqs.(6)-(8) or (21) with $l=10^{-6}$ and $D=10^{-9}$. The distance $r$ in galactic halo region is taken in the range $100-300 \mathrm{Kpcs}$. The values of $\omega$ are negative indicating repulsion.

Fig.4. Plot of $\omega(r)$ vs $r$ in which $\omega$ is computed from either Eqs.(6)-(8) or (21) with $l=10^{-6}$ and $D=0$. The distance $r$ in galactic halo region is taken in the range $100-300 \mathrm{Kpcs}$. The values of $\omega$ are negative indicating repulsion, similar to that in Fig.3.

\section{References}

[1] S. Fay, Astron. Astrophys. 413, 799 (2004)

[2] T. Matos, F.S. Guzmán and D. Nuñez, Phys. Rev. D 62, 061301 (2000)

[3] U. Nucamendi, M. Salgado and D. Sudarsky, Phys. Rev. D 63, 125016 (2001)

[4] F. Rahaman, M. Kalam, A. DeBenedictis, A.A. Usmani and Saibal Ray, Mon. Not. R. Astron. Soc. 389, 27 (2008)

[5] T. Matos, F.S. Guzmán, Class. Quantum Grav. 18, 5055 (2001)

[6] M. Alcubierre, F.S. Guzmán, T. Matos, D. Nuñez, L.A. Ureña-López and

P. Wiederhold, Class. Quantum Grav. 19, 5017 (2002)

[7] F.S. Guzmán and L.A. Ureña-López, Phys. Rev. D 68, 024023 (2003)

[8] F.S. Guzmán and L.A. Ureña-López, Astrophys. J. 645, 814 (2006)

[9] A. Bernal and F.S. Guzmán, Phys. Rev. D 74, 103002 (2006)

[10] S. Chandrasekhar, Mathematical Theory of Black Holes (Oxford University Press, 1983)

[11] A. Boriello and P. Salucci, Mon. Not. R. Astron. Soc. 323, 285 (2001)

[12] S. Bharadwaj and S. Kar S, Phys. Rev. D 68, 023516 (2003)

[13] D. Lynden-Bell, J. Katz and J. Bičák, Phys. Rev. D 75, 024040 (2007)

[14] K.K. Nandi, Y.Z. Zhang, R.G. Cai and A. Panchenko, Phys. Rev. D 79, $024011(2009)$

[15] K.K. Nandi, A. Islam and J. Evans, Phys. Rev. D 55, 2497 (1997)

[16] K.K. Nandi, B. Bhattacharjee, S.M.K. Alam and J. Evans, Phys. Rev. D $\mathbf{5 7}, 823(1998)$

[17] T. Faber and M. Visser, Mon. Not. R. Astron. Soc. 372, 136 (2006) 